\title{
Determination of the coronal magnetic field by coronal loop oscillations
}

\author{
V. M. Nakariakov ${ }^{1}$ and L. Ofman ${ }^{2}$ \\ 1 Physics Department, University of Warwick, Coventry CV4 7AL, UK \\ 2 CUA/NASA Goddard Space Flight Center, 682, Greenbelt, MD 20771, USA \\ e-mail: leon.ofman@gsfc.nasa.gov
}

Received 30 October 2000 / Accepted 26 April 2001

\begin{abstract}
We develop a new method for the determination of the absolute value of the magnetic field strength in coronal closed magnetic structures, based on the analysis of flare-generated oscillations of coronal loops. Interpretation of the oscillations observed in terms of global standing kink waves allows to connect the period of the oscillations and the loops length with the magnetic field strength in the loops. For loop oscillations observed with TRACE on 14th July 1998 and 4th July 1999, we estimate the magnetic field strength as 4-30 G. Using TRACE $171 \AA$ and $195 \AA$ images of the loop, taken on 4th July 1999 to determine the plasma density, we estimate the magnetic field in the loop as $13 \pm 9 \mathrm{G}$. Improved diagnostic of the loop length, the oscillation period, and the plasma density in the loop will significantly improve the method's precision.
\end{abstract}

Key words. magnetohydrodynamics (MHD) - waves - Sun: corona - Sun: activity - Sun: oscillations - Sun: UV radiation

\section{Introduction}

Determination of the coronal magnetic field, the parameter which controls the dynamics, structure and evolution of the corona of the Sun, has been a challenge for solar physicists for several decades. Main efforts have been connected with application of radio methods based on gyroresonant emission (e.g. Schmelz et al. 1994; Brosius et al. 1997; Lee et al. 1999). However, the radio methods are mainly used for the detection of super-strong, few hundred Gauss magnetic fields over active regions. Also, the spatial resolution, $>5^{\prime \prime}$ (e.g. Lee et al. 1999) is low compared to the resolution of modern EUV imaging telescopes TRACE $\left(0.5^{\prime \prime}\right)$ and SOHO/EIT $\left(2.5^{\prime \prime}\right)$.

An alternative method is the analysis of optical and EUV coronal emission spectrum modification due to the Zeeman and Hanle effects, which provides better spatial resolution and in principle allows to detect magnetic fields of the quite Sun magnitudes (see, e.g. Judge 1998 for the discussion). Very recently, Lin et al. (2000) measured the line-of-sight component of the magnetic field using the near-infrared emission line $10747 \AA$ and got $10 \pm 0.5 \mathrm{G}$ and $33 \pm 0.7 \mathrm{G}$ field strength in two different out-oflimb active regions. However, the necessity to reduce the noise level required to reduce drastically the spatial and

Send offprint requests to: V. M. Nakariakov, e-mail: valery@astro.warwick.ac.uk temporal resolution (e.g. the signal integration time was about 70 min with the resolution of about $1^{\prime}$ ).

Faraday rotation observations of polarized radiation from natural radio sources (e.g., Mancuso \& Spangler 2000 and references therein), can work well in the upper part of the corona (e.g., 5-14 solar radii), but fail near the limb.

Indirect methods for the determination of the coronal magnetic field include extrapolation of photospheric magnetic sources (e.g. Cuperman et al. 1990; McClymont et al. 1997) which are measured with the Zeeman effect in photospheric lines. However, this approach has a number of theoretical difficulties and ambiguities.

Recent high resolution observations of MHD wave activity in the corona reported by Aschwanden et al. (1999), Nakariakov et al. (1999) and Schrijver \& Brown (2000) allows us to develop a new method for the measurement of the coronal magnetic field by using MHD coronal seismology. The idea of the method is very similar to the helioseismology: observationally, we can determine properties of coronal waves and oscillations (amplitudes, temporal and spatial spectra, typical signatures and evolution), as well as some physical parameters of the medium supporting the wave propagation (e.g. temperature, density, etc.). The MHD wave theory provides us with formulae connecting the wave properties together with the measured coronal physical parameters (density, temperature, structuring) with the unknown magnetic field strength, and transport coefficients (see, also, Nakariakov 2000). 
Consequently, we can determine the unknown physical parameters of the corona.

The main advantages of the MHD coronal seismology with the EUV imaging telescopes over other methods are: (1) the precise relation of the measurement to a specific coronal structure, and consequent high spatial resolution (less than $\left.1^{\prime \prime}\right),(2)$ both on-the-limb and off-limb measurements are possible, (3) determination of the absolute value of the coronal magnetic field strength that includes all three components.

In this letter, we discuss the possibility for determination of the Alfvén speed and magnetic field through the analysis of flare-generated coronal loop oscillations observed with TRACE on 14th of July, 1998 and 4th of July, 1999.

\section{Observations of flare-generated loop oscillations}

\subsection{4 th of July, 1998}

The movies generated with $171 \AA$ bandpass TRACE images taken on 14th of July, 1998 (Aschwanden et al. 1999; Nakariakov et al. 1999) show kink-like decaying quasiperiodic displacement of several coronal loops shortly after a flare (at 12:11 UT, in the adjacent active region AR8270). The observational sequence with the cadence time of about $75 \mathrm{~s}$ and the exposure time of about $16.4 \mathrm{~s}$ included all stages of the oscillations. The analysis of the intensity variation in four neighbouring perpendicular slits near the loop apex showed synphase transversal displacement. Considering the averaged loop displacement as a function of time and approximating the observationally determined dependence with an exponentially decaying harmonic function, the period of the oscillations was found to be about $\sim 256$ s (Nakariakov et al. 1999). The distance between the loop footpoints was estimated as $\sim 8.3 \times 10^{9} \mathrm{~cm}$, which, for a semi-circular loop gives the loop length of $\sim 1.3 \times 10^{10} \mathrm{~cm}$.

\subsection{4th of July, 1999}

The second example of the flare-generated loop oscillations, reported by Schrijver \& Brown (2000), was less complete. The flare was occurring at 8:20 UT, 14th of July, 1999, when TRACE was passing through the South Atlantic Anomaly. Unfortunately, the TRACE $171 \AA$ observations had a gap between 8:17 and 8:33 UT and only the very last stage of the loop oscillations was registered. Figure 1 shows the temporal evolution of the intensity across an oscillating loop. Three neighbouring slits taken near the loop apex behave almost identically, suggesting that we deal with the kink oscillations. For the elapsed time greater then $600 \mathrm{~s}$ the loop displacement is not seen. It is possible to roughly estimate the period of the decaying oscillations, assuming that we observe one full cycle. The estimation gives the period of $\sim 360 \mathrm{~s}$. The distance between the footpoints is $\sim 330$ pixels. For the pixel size

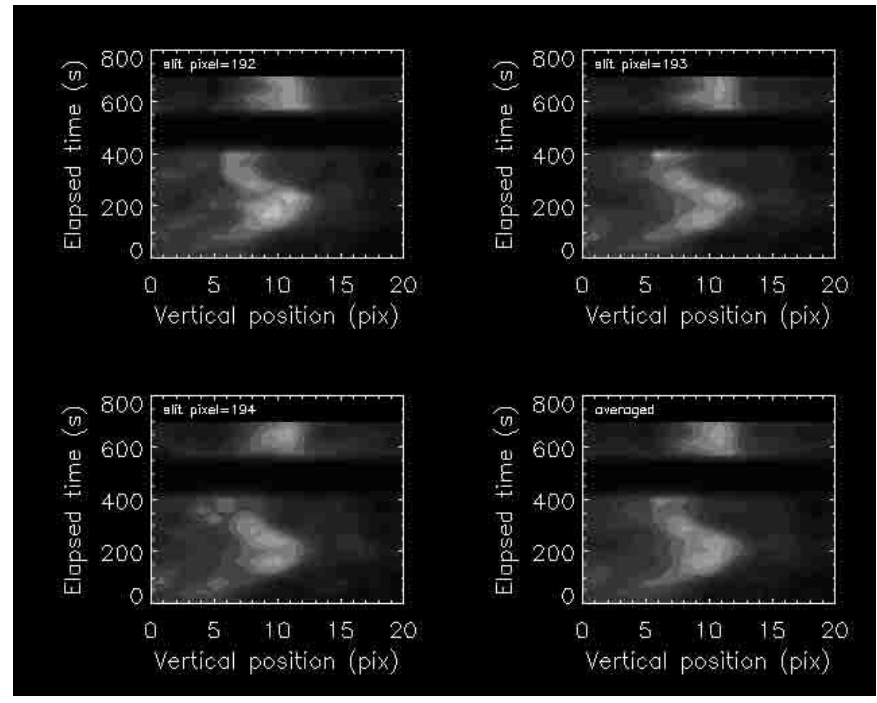

Fig. 1. Evolution of the emission intensity across a coronal loop for the event on July 4th, 1999. The observation starts at 8:33 UT.

$\sim 360 \mathrm{~km}$, it gives the loop diameter along the major axis of $\sim 1.2 \times 10^{10} \mathrm{~cm}$. Assuming the loop is semi-circular, we obtain the loop length $\approx 1.9 \times 10^{10} \mathrm{~cm}$.

\subsection{Global kink modes}

The natural interpretation of these phenomena is that the loops experience kink global mode MHD oscillations. As the main amplitude of the displacement is observed near the loop apecis and the displacement is synphase, we conclude that the oscillations are the global standing mode of the loop, with the wave length double the loop length. Taking the observed periods $P$ and loop lengths $L\left(256 \mathrm{~s}\right.$ and $1.30 \times 10^{10} \mathrm{~cm}$ for 14th of July, $1998 ; 360 \mathrm{~s}$ and $1.9 \times 10^{10} \mathrm{~cm}$ for 4 th of July, 1999), we estimate the phase speed required as

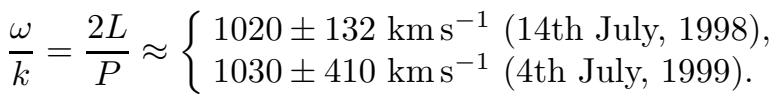

Possible errors of these measurements are discussed in Sect. 5.

\section{MHD modes of coronal loops}

The theory of MHD modes of magnetic structures is well developed (see, e.g. Roberts 1991). Considering a coronal loop as a straight magnetic cylinder of width $a$, one can connect properties of MHD modes of the cylinder with physical conditions inside and outside the cylinder through the dispersion relation:

$$
\begin{aligned}
& \rho_{\mathrm{e}}\left(\omega^{2}-k^{2} C_{\mathrm{Ae}}^{2}\right) m_{0} \frac{I_{\mathrm{m}}^{\prime}\left(m_{0} a\right)}{I_{\mathrm{m}}\left(m_{0} a\right)} \\
& +\rho_{0}\left(k^{2} C_{\mathrm{A} 0}^{2}-\omega^{2}\right) m_{\mathrm{e}} \frac{K_{\mathrm{m}}^{\prime}\left(m_{\mathrm{e}} a\right)}{K_{\mathrm{m}}\left(m_{\mathrm{e}} a\right)}=0,
\end{aligned}
$$


where

$m_{\alpha}^{2}=\frac{\left(k^{2} C_{\mathrm{s} \alpha}^{2}-\omega^{2}\right)\left(k^{2} C_{\mathrm{A} \alpha}^{2}-\omega^{2}\right)}{\left(C_{\mathrm{s} \alpha}^{2}+C_{\mathrm{A} \alpha}^{2}\right)\left(k^{2} C_{\mathrm{T} \alpha}^{2}-\omega^{2}\right)}$,

where $\omega$ and $k$ are the frequency and the longitudinal wave number, respectively; the indices $\alpha=0$, e are for internal and external media, respectively; $I_{\mathrm{m}}(x)$ and $K_{\mathrm{m}}(x)$ are modified Bessel functions of order $m$; the prime denotes the derivative of a function with respect to its argument. For the trapped modes, which are evanescent outside the tube, the condition $m_{\mathrm{e}}>0$ has to be fulfilled. The number $m$ determines the mode structure, and for the kink modes considered $m=1$. In addition, there exist a dispersionless torsional Alfvén mode propagating with the Alfvén speed.

In the observational examples mentioned above, only the global mode was seen, with the longitudinal wavelength of double the loop lengths. For the observed loop widths which are much smaller then loop lengths, it makes $k a \ll 1$, which allows us to simplify the dispersion relation.

There are two kink $(m=1)$ modes in the limit $k a \ll 1$ : slow and fast. The slow kink mode has the phase speed about

$\frac{\omega}{k} \approx C_{\mathrm{T} 0} \equiv \frac{C_{\mathrm{s} 0} C_{\mathrm{A} 0}}{\left(C_{\mathrm{s} 0}^{2}+C_{\mathrm{A} 0}^{2}\right)^{1 / 2}}$.

The phase speed of the fast kink mode is

$\frac{\omega}{k} \approx C_{\mathrm{k}} \equiv\left(\frac{2}{1+\rho_{\mathrm{e}} / \rho_{0}}\right)^{1 / 2} C_{\mathrm{A} 0}$,

where $C_{\mathrm{k}}$ is a so-called kink speed.

\section{Determination of the magnetic field}

The speed of slow waves, $C_{\mathrm{T} 0}$ is below both the Alfvén $C_{\mathrm{A} 0}$ and the sound $C_{\mathrm{s} 0}$ speeds. For the coronal loop temperature of a few $10^{6} \mathrm{~K}$, we estimate the sound speed as $C_{\mathrm{s} 0}\left[\mathrm{~km} \mathrm{~s}^{-1}\right]=152 T^{1 / 2}\left[10^{6} \mathrm{~K}\right]<300 \mathrm{~km} \mathrm{~s}^{-1}$, we conclude that $C_{\mathrm{T} 0}<300 \mathrm{~km} \mathrm{~s}^{-1}$ and, consequently, the slow mode has to be excluded from the consideration. However, the fast wave can have the required phase speed, and it was concluded (Nakariakov et al. 1999) that we observed the standing global kink fast magnetoacoustic mode. The third MHD wave mode, the Alfvén one, is in this geometry a torsional wave confined to the small scale of the loops' cross-section, and cannot be detected by TRACE.

Expression for the kink speed (5) contains two unknown parameters, the Alfvén speed $C_{\mathrm{A} 0}$ and the density ratio $\rho_{\mathrm{e}} / \rho_{0}$. Considering the density ratio as a parameter, we can determine the Alfvén speed in the loop. Fortunately, this dependence is weak and it allows us to minimize the uncertainty in the density ratio. For the quite wide range of possible density ratios, from 0 to 0.3 , the maximum error in determination of the Alfvén speed is just $7 \%$. Assuming $\rho_{\mathrm{e}} / \rho_{0}=0.1$, we obtain $C_{\mathrm{A}}=$ $756 \pm 100 \mathrm{~km} \mathrm{~s}^{-1}$ for the kink speed of $1020 \pm 132 \mathrm{~km} \mathrm{~s}^{-1}$, for the event on 14th July, 1998.

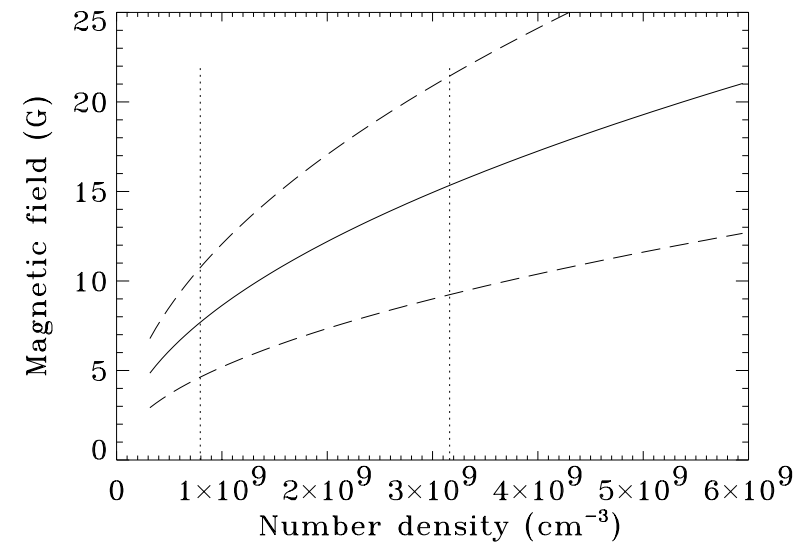

Fig. 2. The magnetic field inside a coronal loop as function of plasma density inside the loop. The external to internal density ratio is 0.1 . The solid curve corresponds to the central value of the kink speed $C_{\mathrm{k}}=1030 \pm 410 \mathrm{~km} \mathrm{~s}^{-1}$ (for the event of the 4 th of July, 1999), and the dashed curves correspond to the upper and the lower possible values of the speed. The vertical dotted lines give the limits of the loop density estimation using TRACE $171 \AA$ and $195 \AA$ images.

The Alfvén speed is defined by the magnetic field strength and the density of the medium. Consequently, we can estimate the value of the magnetic field in the loop:

$B_{0}=\left(4 \pi \rho_{0}\right)^{1 / 2} C_{\mathrm{A} 0}=\frac{\sqrt{2} \pi^{3 / 2} L}{P} \sqrt{\rho_{0}\left(1+\rho_{\mathrm{e}} / \rho_{0}\right)}$.

The determination of the magnetic field is weakly sensitive to errors in the determination of the density, because the magnetic field is proportional to the square root of the density. Figure 2 shows the dependence of the magnetic field on the density for different values of the kink speed and shows that for a quite wide range of plasma densities, from $1 \times 10^{9}$ to $6 \times 10^{9} \mathrm{~cm}^{-3}$, the magnetic field is in the range from 4 to $30 \mathrm{G}$.

We have used TRACE $171 \AA$ and $195 \AA$ images of the loop taken on July 4th, 1999 at about 8:41 UT to determine the loop's temperature and emission measure using the TRACE data analysis routines. We found that the loops' temperature is about $1.01 \times 10^{6} \mathrm{~K}$ near the loop top, and about $1.05 \times 10^{6} \mathrm{~K}$ midway between the loop top and the flare site. We have used the emission measure at these location to determine the loop density assuming that the loop width is 4 pixels $\left(1.44 \times 10^{8} \mathrm{~cm}\right)$ and the filling factor is 1.0, and found that the density is $10^{9.3 \pm 0.3} \mathrm{~cm}^{-3}$ at the loop top and $10^{9.2 \pm 0.3} \mathrm{~cm}^{-3}$ in the midway section. According to the TRACE Data Analysis Guide (Bentley, R.D., 2000, http://umbra.nascom.nasa.gov/bentley/guides/tag/) the emission measure determined with this technique is known to a factor of 4 . Thus the above values of the density are determined to a factor of two, which produces the error bar of 0.3 in the exponent. Using the above values of the density we get the magnetic field in the range $B=13 \pm 9 \mathrm{G}$ (see Fig. 2). Future improvement in the accuracy of the density determination will provide better estimates of the field with this technique. 


\section{Error analysis}

Our method allows to determine the absolute value of the magnetic field strength in a coronal loop, measuring the period of the global kink mode, the loop length, the plasma density inside the loop, and the ratio of the densities inside and outside the loop. According to Eq. (6), the main sources of uncertainty in the determination of the coronal magnetic field are the uncertainties in the period of oscillations and the loop length, while the effect of uncertainty in the measurement of the densities is dramatically reduced by the square root dependence.

Estimating the errors in the determination of the loop length, we conclude that it is mainly due to projection effects and the departure of the loop shape from circular. However, it may hardly exceed 10-15\%. Indeed, assuming that the shape of the loop is elliptic with the principal axis length $R$ and the eccentricity $e$, the $15 \%$ difference between the circle semi-length $(\pi R)$ and the ellipse semi-length $\left(\approx \pi R\left(1-e^{2} / 4\right)\right)$ occurs for the eccentricity $e \approx 0.77$. The error in the determination of the oscillation period is sensitive to the condition of the specific observation.

In particular, in Nakariakov et al. (1999), the error of the period was just $3 \%$ because several oscillating cycles were observed. In the event analyzed in Sect. 2.2 (see, also Schrijver \& Brown 2000), the error is probably much larger, about $30 \%$. The accuracy of determination of the densities depends upon the specific method applied and is about 50\% (e.g. Mason et al. 1999 and references therein). The determination of the density ratio possibly has even larger error, but as the ratio $\rho_{\mathrm{e}} / \rho_{0}$ is assumed to be small, one can neglect its value in Eq. (6). Thus, the relative error of the method can be estimated as

$\delta B_{0}=\sqrt{(\delta L)^{2}+(\delta P)^{2}+\left(\delta \rho_{0} / 2\right)^{2}}$.

For example, for relative errors in the loop length $\delta L \approx$ $10 \%$, oscillation period $\delta P \approx 3 \%$ and density $\delta \rho_{0} \approx 50 \%$, the relative error in the magnetic field determination $\delta B_{0}$ can be reduced below $30 \%$.

\section{Conclusions}

In this letter we demonstrate that the method of MHD coronal seismology with EUV imaging telescopes, can be used for the determination of coronal magnetic field in fine structures of the solar corona. Further development and application of the method requires effort in both, observational and theoretical directions.

Observationally, it is important to search for new examples of flare generated coronal loop oscillations in TRACE data. The incidence of this rare phenomena should increase during solar maximum, and indeed several new cases of coronal loop oscillations were detected by TRACE in March and April 2001. Also, measurement of the density in oscillating coronal loops is needed. In particular, joint observational campaigns with high resolution imaging telescopes and spectral instruments, of density sensitive emission lines are required to better determine the magnetic field strength. In particular, development of high resolution spectral observations of the corona (e.g. O'Shea et al. 2001) seems to be very promising.

Theoretically, it is necessary to develop a model of coronal loop oscillations, taking into account effects of dissipation, gravitational density stratification, curvature, divergence, and twist of the loop's magnetic field, as well as nonlinear effects connected with finite displacement of the loop. More specifically, the impact of these effects on the kink speed, the oscillation period, and the decay rate of the oscillations needs to be investigated.

Acknowledgements. The authors are grateful to Daniel Brown for the provided data on the event of 14th of July, 1999. LO would like to thank the NASA SR\&T Program (NASW-98004) and the NASA Sun-Earth Connection Theory Program. Part of this work was performed while LO was visiting at Tel Aviv University. Also, the authors are grateful to the referee, Markus Aschwanden, for helpful comments.

\section{References}

Aschwanden, M. J., Fletcher, L., Schrijver, C. J., \& Alexander, D. 1999, ApJ, 520, 880

Brosius, J. W., Davila, J. M., Thomas, R. J., \& White, S. M. 1997, ApJ, 488, 488

Cuperman, S., Ofman, L., \& Semel, M. 1990, A\&A, 230, 193

Judge, P. G. 1998, ApJ, 500, 1009

Lee, J., White, S. M., Kundu, M. R., Mikić, Z., \& McClymont, A. N. 1999, ApJ, 510, 413

Lin, H., Penn, M. J., \& Tomczyk, S. 2000, ApJ, 541, L83

Mancuso, S., \& Spangler, S. R. 2000, ApJ, 539, 480

Mason, H. E., Landi, E., Pike, C. D., \& Young, P. R. 1999, Solar Phys., 189, 129

McClymont, A. N., Jiao, L., \& Mikic, Z. 1997, Solar Phys., 174,191

Nakariakov, V. M., Ofman, L., DeLuca, E. E., Roberts, B., \& Davila, J. M. 1999, Science, 285, 862

Nakariakov, V. M. 2000, in Waves in Dusty, Solar and Space Plasmas, ed. F. Verheest et al. (AIP CP537, New York)

O'Shea, E., Banerjee, D., Doyle, J. G., Fleck, B., \& Murtagh, F. 2001, A\&A, 368, 1095

Roberts, B. 1991, in Advances in Solar System Magnetohydrodynamics, ed. E. R. Priest, \& A. W. Hood (CUP, Cambridge), 105

Schmelz, J. T., Holman, G. D., Brosius, J. W., \& Willson, R. F. 1994, ApJ, 434, 786

Schrijver, C. J., \& Brown, D. S. 2000, ApJ, 537, L69 